\title{
Preparing healthcare facilities to operate safely and effectively during the COVID-19 pandemic: The missing piece in the puzzle
}

\author{
L S Wilkinson, ${ }^{1,2} \mathrm{MSc} ;$ S Moosa, ${ }^{3} \mathrm{MMed}, \mathrm{MBA}, \mathrm{PhD}$; R Cooke, ${ }^{3}$ BBusSci (Finance), MB BCh, MMed (Family Medicine); \\ A M Muller, ${ }^{4,5}$ MB ChB, MRCGP (Lond), Dip HIV Man (SA); T H Boyles, ${ }^{6}$ BM BCh, MRCP, MD, DTM\&H, Cert Infectious Diseases (SA) \\ ${ }^{1}$ International AIDS Society, Johannesburg, South Africa \\ ${ }^{2}$ Centre for Infectious Epidemiology and Research, Faculty of Health Sciences, University of Cape Town, South Africa \\ ${ }^{3}$ Department of Family Medicine, Faculty of Health Sciences, University of the Witwatersrand, Johannesburg, South Africa \\ ${ }^{4}$ Nkqubela Tuberculosis Hospital, Department of Health, Eastern Cape, South Africa \\ ${ }^{5}$ Rural Doctors Association of South Africa \\ ${ }^{6}$ Department of Medicine, Faculty of Health Sciences, University of Cape Town, South Africa
}

Corresponding author: L S Wilkinson (lynne.susan.wilkinson@gmail.com)

\begin{abstract}
The stated objective of the COVID-19 lockdown was to allow time to prepare healthcare facilities. Preparation must include administrative and environmental measures, which when combined with personal protective equipment, minimise the risk of the spread of infection to patients and healthcare workers (HCWs) in facilities, allowing HCWs to safely provide essential services during the pandemic and limit the indirect effects of COVID-19 caused by healthcare disruption. We present our model for facility preparation based on colour-coded zones, social distancing, hand hygiene, rapid triage and separate management of symptomatic patients, and attention to infection transmission prevention between HCWs in communal staff areas. This model specifically addresses the challenges in preparing a facility for COVID-19 in a low-resource setting and in rural areas. In addition, we include links to resources to allow workers in low-resource settings to prepare their facilities adequately.
\end{abstract}

S Afr Med J 2020;110(9):835-836. https://doi.org/10.7196/SAMJ.2020.v110i9.15094

When the scale of the COVID-19 pandemic became evident, many countries introduced lockdowns with the stated aim of buying time to prepare healthcare facilities. Most efforts have focused on developing increased capacity to care for confirmed cases, such as buying ventilators and constructing field hospitals, along with the necessary procurement of personal protective equipment (PPE) to prevent transmission of infection from patients to healthcare workers (HCWs). Less attention has been given to the arguably more important aim of preventing transmission of COVID-19 between HCWs and patients in healthcare facilities, including primary care outpatient clinics.

Preventing infections in healthcare facilities is uniquely difficult and important. Healthcare facilities are areas of mass gathering that cannot be closed during a lockdown. By their nature, they attract people who are sick and may have COVID-19, as well as people with comorbidities, who are most at risk of severe disease if infected. In addition, staff members are at increased risk of infection due to heightened exposure.

While failure to implement an effective primary care COVID-19 preparedness approach can have direct effects due to an increased incidence of COVID-19, the consequences of viral transmission within facilities are much broader, as service delivery can be severely compromised when many staff members are infected, and attendance will drop if patients are afraid to attend facilities because of fear of becoming infected. This effect was profound during the Ebola outbreak in West Africa in 2014/15, ${ }^{[1]}$ and a recent modelling study predicted 1157000 additional child deaths and 56700 additional maternal deaths over 6 months in low- and middle-income countries because of health system disruption due to COVID-19. ${ }^{[2]}$
When the lockdown began in South Africa (SA) on 27 March 2020, and based initially on our experience of preparing facilities during the Ebola outbreak in West Africa in 2014/15, we developed a series of resources focused on administrative, environmental and PPE measures to allow healthcare facilities to function safely and effectively during the COVID-19 pandemic. Since then we have provided technical assistance to over 150 primary healthcare facilities in SA and updated the resources based on our experience. Here we present our methods along with links to the technical resources.

\section{Methods}

Our approach divides clinics and district hospitals into three colourcoded zones based on COVID-19 risk. The yellow zone (intermediate risk) begins with the clinic queue and ends when patients are screened for symptoms. Patients who screen positive enter the orange zone (high risk), and those who screen negative enter the blue zone (low risk). Brightly coloured posters and floor paint guide patients through the applicable service delivery zones in the facility and end at separate orange and blue exits. A key principle is that once screened, patients do not cross paths with patients in separate zones.

Public sector healthcare facilities in low-resource settings tend to see patients on a first-come, first-served basis. It is therefore common for large numbers to begin congregating outside clinic gates well before dawn. Facility preparedness therefore begins with taking control of the area outside the clinic by ensuring a controllable single point of entry and painting lines $1.5 \mathrm{~m}$ apart for as far as is necessary to ensure that the queue is socially distanced. Patients are then empowered to adhere to social distancing rules without fear of missing their place in the queue. Some clinics have adopted separate 
queues for priority groups such as women and children seeking maternal and child health services. A queue marshal manages the line and educates patients about changes to service provision that can be expected upon entry, in particular reassuring them that should they report their COVID-19 symptoms, they will receive all the services for which they are attending in a designated area in the facility and will not be required to join any further queues for services.

After passing through the single point of entry one at a time, patients receive hand sanitiser from a designated officer and their cloth mask is checked for correct fit. They then move to the last yellow zone station - the first screening station, where nonprofessional HCWs (nursing assistants, community health workers or volunteer health science students) rapidly triage them into COVID19 symptom positive and negative. Screening takes place outside the facility building. Being outdoors is essential because good ventilation is a known defence against the spread of the virus, and it reduces the number of people inside the clinic at any time. Screening questions (see Box 1) are intended to be highly sensitive to triage any person at risk into the orange zone.

The orange zone, for high-risk symptomatic patients, is also typically set up outside the clinic building under gazebos or in larger tents, although isolated areas in clinic buildings can be used for some or all orange zone stations, if they have a dedicated entrance. Patients remain in the orange zone throughout their clinic visit and carry their chair from station to station to prevent cross-infection. Their first station is the orange zone clinician, who assesses disease severity and deals with COVID-19 symptoms, including requests for HIV and tuberculosis (TB) testing as appropriate. Severity is evaluated by assessing oxygen saturation, respiratory rate and temperature, so a portable or mobile fingertip oximeter is required. A runner fetches files and prescribed medicines from inside the main clinic building if necessary. The clinician also attends to any other needs of the patient, such as collection of chronic medication, antenatal care, immunisations or non-COVID-19 symptoms. If the patient requires attention that is beyond the scope of practice of the clinician (e.g. a complicated antenatal visit), a separate clinical area is available in the orange zone for a specialised clinician (such as a midwife) to come from the blue zone (routine services) to attend to the patient. The orange zone also includes an isolation room or gazebo with an examination bed and oxygen for any patient who is awaiting patient transport to a referral hospital. Facilities have repurposed outside HIV testing containers, storage, waste management and other rooms, or alternatively set up a gazebo. In addition, there are gazebos or screened-off spaces for HIV testing and collection of both TB and COVID-19 specimens. The patient then leaves the facility by a separate exit point, leaving their chair for disinfection and reallocation, having received all services but without interacting with low-risk patients. Increased focus is placed on surface disinfection throughout the orange zone.

Patients with COVID-19 symptoms may need to enter specialist areas of the clinic. Typically, unstable patients go to the emergency department after hours and women in labour to the obstetric unit. Each department must designate a separate orange zone where they can attend to these patients safely.

Patients who are negative for COVID-19 symptoms proceed to the blue zone in the clinic building. Precautions to prevent transmission are still important, as there are likely to be patients and staff members with presymptomatic or asymptomatic disease. Patients follow a oneway route to attend required services. In the blue zone, every second chair or space on a bench is blocked off to ensure social distancing. At each station, patients are called by name or number rather than shifting from chair to chair to get to the front of a queue.
Box 1. In the past 14 days, have you developed ANY of the following:

- Cough

- Fever

- Shortness of breath

- Sore throat

- Loss of sense of smell or taste

- Worsening of a chronic cough

Transmission of COVID-19 between staff members is emerging as a significant risk to continued services. All staff are required to selfscreen at home and are rescreened for symptoms on arrival, with symptomatic staff being advised to self-isolate at home. Particular attention is given to communal staff rooms such as tearooms and offices. There is strict social distancing, hand hygiene and mask wearing, and a maximum number of people are allowed in each room at any given time. Lunch and tea breaks are staggered or held outside, or even in individuals' cars. Staff are also educated on the risks of transmission outside the facility, particularly when sharing public or private transport to and from work.

The administrative and environmental adaptations that we describe will only be effective if accompanied by appropriate use of PPE, hand hygiene and environmental cleaning, which are included in our training material but are beyond the scope of this article.

Our experience has been that clinics make great efforts to prepare for COVID-19 but require in-person technical assistance in order to optimise these efforts. We have typically visited a clinic two or three times to technically support set-up and briefing on the clinical management algorithm for symptomatic patients managed in the orange zone, then continued to provide support on an ad hoc basis. It has been important to show flexibility to the needs of particular clinics and adapt our strategies accordingly. We have developed a series of guides, posters and videos to accompany this work. The links to all the training materials are available (http:// www.differentiatedcare.org/Resources/Resource-Library/COVID19-DSD-Resources-Health-facility-preparedness).

\section{Discussion}

Preparation of healthcare facilities to prevent cross-infection of COVID-19 is vitally important to avoid direct and indirect harms to healthcare service delivery. The measures we have outlined adhere to national COVID-19 clinical and infection prevention and control guidance and have proved practical to implement in primary healthcare facilities in SA. We encourage HCWs in other low-resource settings to implement these measures, utilising the resources provided.

Declaration. None.

Acknowledgements. None.

Author contributions. Approach development including resources: LW, TB. Approach implementation and review: LW, TB, SM, RC, AMM. Writing, original draft: LW, TB. Writing, review and editing: LW, TB, SM, RC, AMM. Funding. None.

Conflicts of interest. None. 1. Elston JW, Cartwright C, Ndumbi P, Wright J. The health impact of the $2014-15$ Ebola outbreak.
Public Health 2017;143:60-70. https://doi.org/10.1016/j.puhe.2016.10.020
2. Roberton T, Carter ED, Chou VB, et al. Early estimates of the indirect effects of the COVID-19
pandemic on maternal and child mortality in low-income and middle-income countries: A modelling
study. Lancet Glob Health 2020;8(7):e901-e908. https://doi.org/10.1016/S2214-109X(20)30229-1

Accepted 6 July 2020 